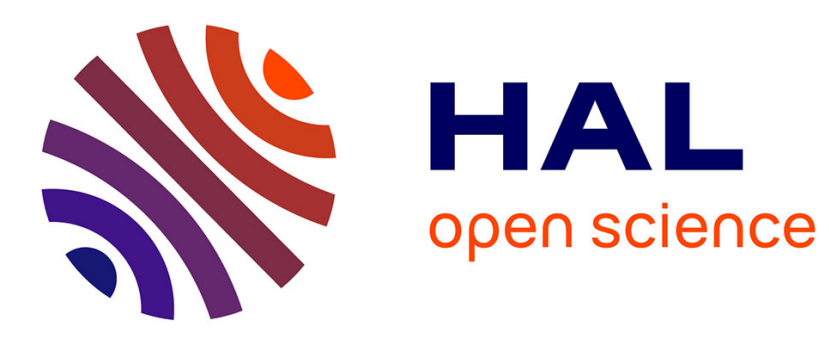

\title{
A Three-Scale Cracking Criterion for Drying Soils
}

Tomasz Hueckel, Boleslaw Mielniczuk, Moulay Saïd El Youssoufi, Liang Bo

$\mathrm{Hu}$, Lyesse Laloui

\section{To cite this version:}

Tomasz Hueckel, Boleslaw Mielniczuk, Moulay Saïd El Youssoufi, Liang Bo Hu, Lyesse Laloui. A Three-Scale Cracking Criterion for Drying Soils. Acta Geophysica, 2014, 62 (5), pp.1049-1059. 10.2478/s11600-014-0214-9 . hal-01141492

\section{HAL Id: hal-01141492 \\ https://hal.science/hal-01141492}

Submitted on 13 Apr 2015

HAL is a multi-disciplinary open access archive for the deposit and dissemination of scientific research documents, whether they are published or not. The documents may come from teaching and research institutions in France or abroad, or from public or private research centers.
L'archive ouverte pluridisciplinaire HAL, est destinée au dépôt et à la diffusion de documents scientifiques de niveau recherche, publiés ou non, émanant des établissements d'enseignement et de recherche français ou étrangers, des laboratoires publics ou privés. 


\title{
A Three-Scale Cracking Criterion for Drying Soils
}

\author{
Tomasz HUECKEL ${ }^{1}$, Boleslaw MIELNICZUK ${ }^{2,3}$, \\ Moulay S. EL YOUSSOUFI ${ }^{3,4}$, Liang B. HU ${ }^{5}$, and Lyesse LALOUI ${ }^{6}$ \\ ${ }^{1}$ Duke University, Durham, USA; e-mail: hueckel@duke.edu \\ ${ }^{2}$ Institut de Radioprotection et de Sûreté Nucléaire, \\ Saint-Paul-lez-Durance, France; e-mail: boleslaw.mielniczuk@univ-montp2.fr \\ ${ }^{3}$ Laboratoire de Micromécanique et d'Intégrité des Structures, IRSN, CNRS, \\ Université de Montpellier 2, Montpellier, France \\ ${ }^{4}$ Laboratoire de Mécanique et Génie Civil de Montpelier, CNRS, Université \\ de Montpellier 2, France; e-mail: Moulay-Said.El-Youssoufi@univ-montp2.fr \\ ${ }^{5}$ University of Toledo, Toledo, USA; e-mail: liangbo.hu@utoledo.edu \\ ${ }^{6}$ École Polytechnique Fédérale de Lausanne (EPFL), Lausanne, Switzerland \\ e-mail: lyesse.laloui@epfl.ch
}

\begin{abstract}
Cracking is a most unwanted development in soil structures undergoing periodic drying and wetting. Desiccation cracks arise in an apparent absence of external forces. Hence, either an internal, self-equilibrated stress pattern resulting from kinematic incompatibilities, or a stress resulting from reaction forces at the constraints appear as a cracking cause, when reaching tensile strength. At a meso-scale, tubular drying pores are considered in the vicinity of a random imperfection, inducing a stress concentration in the presence of significant pore suction. This approach allows one to use the effective stress analysis, which otherwise, away from the stress concentration, usually yields compressive effective stress and hence a physically incompatible criterion for a tensile crack. Recent experiments on idealized configurations of clusters of grains provide geometrical data suggesting that an imperfection as a result of air entry deep into the granular medium penetrates over 4 to 8 internal radii of a typical pore could yield a tensile effective stress sufficient for crack propagation.
\end{abstract}

Key words: drying, cracking, air entry, stress concentration, suction. 


\section{INTRODUCTION}

Cracking is a highly undesirable occurrence in soil structures subject to drying, from both mechanical strength and hydraulic points of view, usually degrading engineering soil quality. Per definition, drying is a multi-phase phenomenon, with a quite complex multi-physics, including phase change, capillarity, flow, deformability, and (perhaps) water cavitation. Drying and drying cracking require considerations to be made at three scales: macroscale continuum, meso-scale of grain and pore clusters, and micro-scale of individual pore structure or grains with liquid bridges.

Drying cracking has for years been marred with a criterion paradox: the tensile drying cracks seem to occur at a compressive effective stress state. The purpose of this paper is to provide a criterion that disentangles the paradox.

Similarly to thermal cracking, the desiccation cracking appears in the apparent absence of external macro-scale forces. Therefore, either an internal, self-equilibrated stress pattern due to kinematic incompatibilities and moisture distribution or a stress resulting from reaction forces at the constraints (or all the above) should be taken into account in a cracking criterion. The following phenomena and conditions need to be considered for drying cracks: drying shrinkage, kinematic constraints impeding the shrinkage and hence inducing reaction forces, and, consequently, tensile effective stress reaching tensile strength.

An earlier tubular micro-scale model of a drying porous medium can be considered in several configurations: unconstrained, completely constrained at the contact with the material matrix, and constrained at restrictive interpore solid contacts. The latter one is addressed in this paper. At the mesoscale, tubular drying pores are considered in the vicinity of an imperfection, inducing a stress concentration near its tip, in the presence of significant pore suction. This approach allows one to use the effective stress analysis, which otherwise, away from the stress concentration, usually yields compressive effective stress and hence a physically incompatible criterion. Experimental results from an idealized configuration of a cluster of grains at micro-scale provide geometrical data suggesting that an imperfection as a result of air entry deep into the granular medium penetrates over 8 internal radii of the typical pore.

\section{THEORETICAL AND EXPERIMENTAL BACKROUND}

Drying and drying cracking require considerations to be made at three scales: macro-scale continuum, at which stress, effective stress, and strength criteria are formulated and soil experiments are conducted; meso-scale of clusters of grains and pores, where structural of air entry effects can be ex- 
perimentally observed and analyzed; and micro-scale of single pore or grain water/vapor, where capillary forces can be observed.

Macroscopic experiments with desiccation on wet soil strip bars indicate that most of the shrinkage during drying takes place when soil is still saturated. Shrinkage stops practically simultaneously with the air entrance into the soil, at the water content value (hence referred to as shrinkage limit) still above $20 \%$ for the tested soils (Péron et al. 2009, 2010, 2013). The remaining drying process occurs with a much-reduced shrinkage rate, but almost entirely via desaturation (Corte and Higashi 1964, Fredlund and Rahardjo 1993, Kodikara et al. 2002, Pellenq et al. 2009). However, specific mechanisms of the shrinkage cessation and air entry and their relationships are still not well understood.

In this context, we examined (Hu et al. 2013a, b) microscopic data of the pore system evolution as represented by the mercury porosimetry results and postulated corresponding alternative mechanisms based on the pressure (suction) development in the pore-vessels and ensuing water cavitation at a critical suction.

The event of the air entry in the former mechanism is interpreted as an external meniscus plunging (Terzaghi 1927), while the latter consists of a subcutaneous cavitation of water at the boundary (Scherer 1992). The two are physically undistinguishable phenomena (Brinker and Scherer 1990). The former suggests a meniscus shrinking until its diameter becomes smaller than the pore entrance. The latter, implying that water suction reaches tensile water strength, depends notoriously on the presence of dissolved air or solid impurities in water. The numerical criteria for both events yield the same values of a critical suction, given the pore-vessel size.

$$
p_{\text {crit }}=-\frac{2 T_{s} \cos \theta}{a}
$$

where $p_{\text {crit }}$ is Laplace's equilibrium capillary pressure at the air entry, $a$ is the pore radius equal to interface curvature radius, while $T_{s}$ is the surface tension, and $\theta$ is the contact angle.

The onset of cracking follows shortly the first air entry event. Based on a stress concentration mechanism previously proposed by Scherer (1992), we suggest that the imperfection (flaw) of the material at which the stress concentration occurs is generated by the air entry (Hu et al. 2013a-d).

Micro-scale experiments with smooth glass grain clusters or pairs (Mielniczuk et al. 2013, 2014) with capillary water between them imply that the air entry constitutes a surface instability of the water body, depending most likely on the local water pressure. 
To bring the above findings together, our multi-scale approach is dictated by an opportunistic use of experimental possibilities and findings, conceptual hypotheses and numerical models and devices that provide a basis for a realistic scenario to model this complex set of phenomena.

\section{CONCEPTUAL FRAMEWORK}

\subsection{Pore suction build-up}

Experiments with drying silt bars filled with three different fluids (water, ethanol, and glycol) pointed out that their drying shrinkage mainly depends on compressibility of the solids and on surface tension of the liquid (Hu et al. 2013c). These findings prompted a micro-scale model (Hu et al. 2013a), in which shrinkage is viewed as a closure of the mercury porosimetry matching bi-modal pore-vessel system (Fig. 1a) during three stages of the process: (i) Poiseuille flow induced (negative) pressure in the entire tube, hence controlled by compressibility of the material at the meso-scale; however, the shrinkage amount and limit is correlated with surface tension of the liquid, which controls all the capillarity phenomena; this indicates that the capillary force at the meniscus at the external surface is a limiting factor in the end of the deformable tube water transport, and the onset of desaturation; (ii) invasion of air followed by the liquid-vapor interface motion with the simultaneous Poiseuille flow in the central portion of the tube until the pressure gradient ceases; followed by (iii) the motion of the liquid-vapor interface as a sole mechanism of water removal, at a constant liquid pressure and deformation.

Viscous flow through a single (straight) microscopic vessel is described by Hu et al. (2013a) in terms of the fluid pressure in the pore-vessel, $p(x, t)$,

$$
\frac{\partial^{2} p}{\partial x^{2}}+\frac{2 a_{0}}{H h\left[1-\frac{a_{0} p}{H h}\right]}\left(\frac{\partial p}{\partial x}\right)^{2}-\frac{16 \mu}{a_{0}} \frac{\left[1-\frac{a_{0} p}{H h}\right]}{H h} \frac{\partial p}{\partial t}=0
$$

The boundary conditions at the tube center and exit are: $x=0$, $\partial p / \partial x=0, x=x_{0}, p=p_{0}(t)$, respectively; $p_{0}(t)$ is the pressure exerted at the point of evaporation, which is at $x=x_{0}$ at the interface between liquid and vapor phase, with $x_{0}(t=0)=L, L$ being the tube half-length. $H$ is the inelastic stiffness modulus (1D), $\mu$ is the fluid viscosity, $h$ is the thickness of the tube, and $a_{0}$ is the tube initial inner radius. Equation 2 is a linearized form of the pressure vessel deformation equation (Fung 1984), under a 1D flow assumption, and of zero Poisson ratio (Fig. 1b). Last displacement limitation does not apply. 
a)

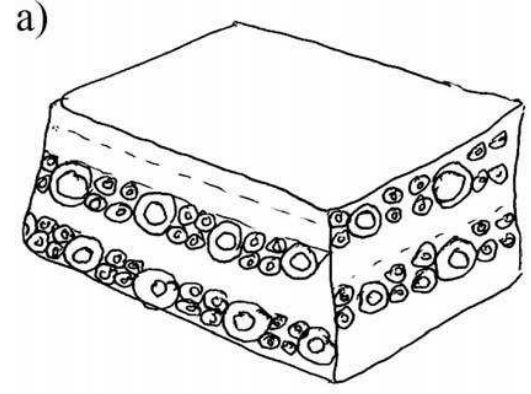

b)

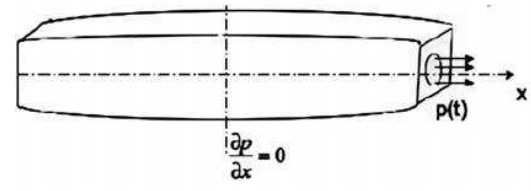

c)

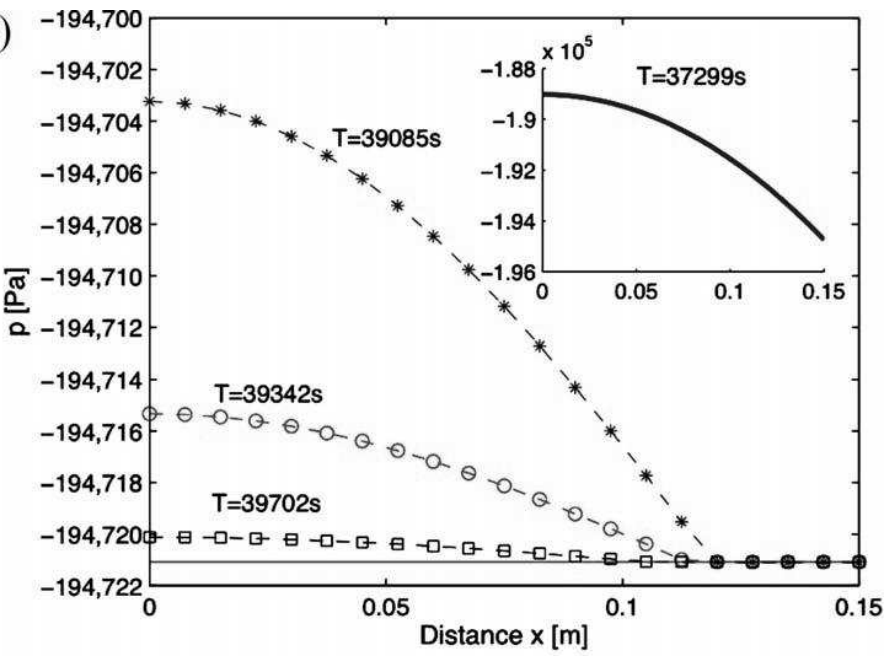

Fig. 1: (a) Two-mode system of cylindrical pore-vessels; (b) individual vessel deformation induced by a pore pressure generated by the external vapor flow; and (c) evaporation flux induced evolution of pore (negative) pressure in pore vessels during flow, with a near-exit segment at a critical pressure and hence instantaneously evaporating. $T$ is time from the onset of evaporation.

The most important product of this micro-scale simulation is the evolution of the pore pressure (Fig. 1c). Visible in the figure is the suction gradient, which responds to the vapor flux at the interface, the displacement of which, starting from the moment of the first entry, becomes the dominant transport mode, given the fact that suction gradient in the liquid phase segment decreases very fast.

\subsection{Air entry}

The above numerical micro-structural studies calibrated against the experimental results of Péron et al. (2007) yield the value of suction corresponding to the air entry. A numerical criterion for the air entry is generally accepted (Terzaghi 1927, see also Lu and Likos 2004) and expressed via critical pressure of the fluid (Eq. 1). The point to be stressed is that the change of pore size during drying is substantial, and hence the value of the critical pore size needs to be accordingly adjusted. The resulting values of the critical pore pressure are $194 \mathrm{kPa}$ for the class of large pores of the order of the original $1.5 \mu \mathrm{m}$ that shrunk to $0.78 \mu \mathrm{m}$, and $280 \mathrm{kPa}$ for the class of small pores of the order of the original $0.5 \mu \mathrm{m}$ that shrunk to $0.42 \mu \mathrm{m}$ (Hu et al. 2013b).

The air entry phenomenon, despite that it occurs at the scale of a single pore, is considered a macroscopic property of soil, mainly based on the assumption of the equality of the fluid pressure at both scales. A suggestive air entry mechanism in a 2D granular system has been proposed by Childs (1969) shown in terms of a succession of stages during drying and re-wetting (Fig. 2). 


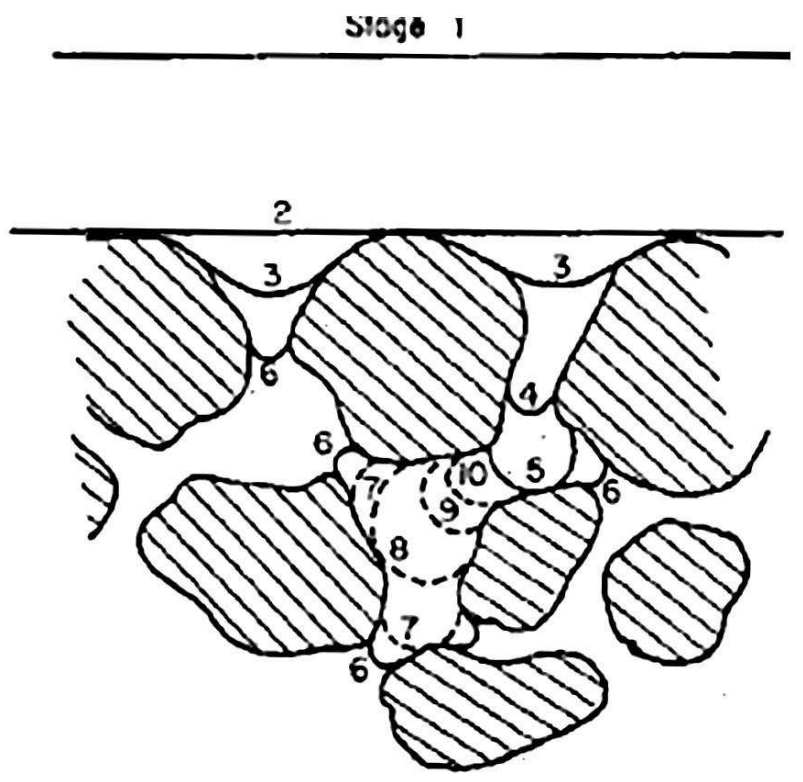

Fig. 2. An early 2D model of air entry into soil (after Childs 1969).

Recently undertaken experiments at a meso-scale with drying of model clusters of smooth glass grains (Mielniczuk et al. 2013) with the funicular water between them reveal new features of the air entry mechanisms. First, it appears that there are multiple modes of air entry. The most characteristic that were identified in all experimental systems tested of 3, 4, 5, 6, and 8 grains were two classes corresponding to a negative or positive local Gaussian curvature (which is a product of the two principal curvatures) of the surface of local meniscus. Most typically, for a negative Gaussian curvature (or concave/convex meniscus) a non-symmetric mode of the unstable evolution of the water body constitutes a most common air entry mechanism into the body of the saturated soil. While the circumstances and numerical criterion for the event are still an open question, it clearly is an unstable and localized displacement of the gas/liquid interface in form of a finger, occurring at a several orders of magnitude higher rate than the preceding process manifested, among others, by a slow evolution of the liquid/gas interface. Figure 3 shows three of a series of images of a 2D saturated cluster of 8 grains (Mielniczuk et al. 2013) shot every 60 seconds during natural isothermal drying (using Canon EOS 500D camera). Grains were $3.5 \mathrm{~mm}$ in diameter ultrafine glass spheres, while the liquid is ultra-pure deionized water. As indicated, it took 10 minutes from the initial configuration to reach the intermediate one, and less than 60 additional seconds to develop the final configuration with the gas penetration. The depth of the penetration of the gas finger is about 4 times the average size (diameter) of the pore. For smaller configurations a fast camera imaging revealed that the unstable process takes about $1 / 2000$-th of a second to develop a new configuration. 
a)

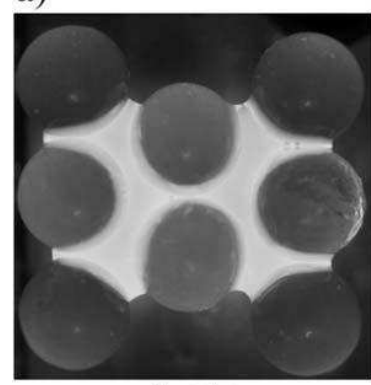

$0 \mathrm{~min}$ b)

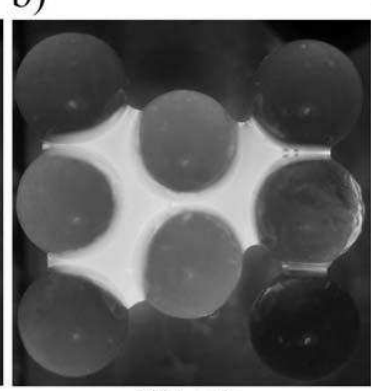

$10 \mathrm{~min}$

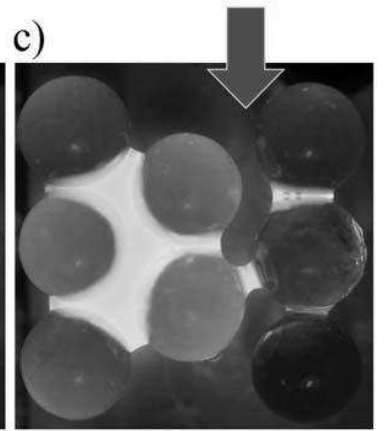

$11 \mathrm{~min}$

Fig. 3. Evolution of the water body between 8 glass spheres subjected to evaporation at a constant temperature and constant ambient vapor pressure. The arrow indicates a localized non-symmetric unstable mode of the interface evolution (air entry finger).

a)

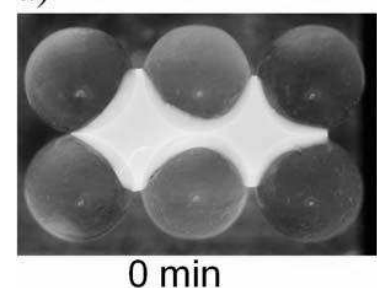

b)

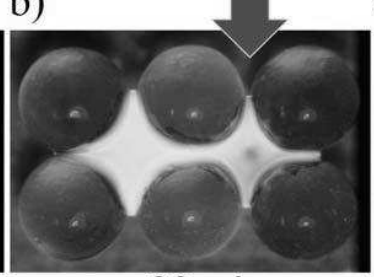

$20 \mathrm{~min}$

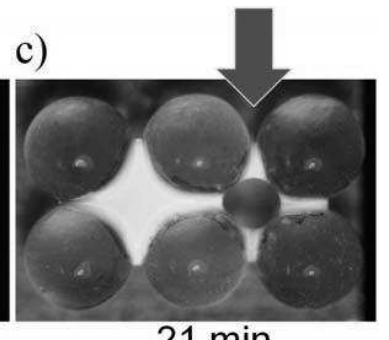

$21 \mathrm{~min}$

Fig. 4. Evolution of the water body between 6 glass spheres subjected to evaporation at a constant temperature and constant ambient vapor pressure. The arrows indicate a localized symmetric unstable mode of the interface evolution (air entry via thin film instability).

The images posterior to the air entry show a wave motion over few seconds of the water mass displaced from the space occupied by the gas finger.

An alternative, symmetric entry mode takes place at a point with a positive Gaussian curvature (concave/concave meniscus) between the spheres and consists of a multi-step process: starting with a convergence of two gas/liquid interfaces situated opposite one another (at the front and back of the picture), followed by formation of a suspended thin film of liquid, followed by a further thinning of the film, leading to coalescence of the two surfaces, producing a short cylindrical or spherical gas nucleus, which subsequently propagates symmetrically with a circular projection until its boundary reaches the solid walls of the sphere. This is shown in Fig. 4 for a six-sphere cluster. Notably, the non-symmetrical scenario occurs for systems with higher separations between grains, while for lower separations a symmetrical scenario takes place. The unstable part of the process starts at the point when the thin film of water bifurcates. The bifurcations of thin films and thin sheets have been known for some time (see, e.g., Taylor 1959), but for capillary bridges have been observed only recently (Maeda et al. 2003). 


\subsection{Drying cracking}

As mentioned earlier, modeling of desiccation cracking via macroscopic approach suffers from a major handicap. Consideration of a 1D drying of a constrained saturated granular body easily shows that a modest total stress is generated in reaction to the constraints and that it is tensile, whereas the values of suction induced by the evaporation flux are significant. Consequently, the resulting effective stress appears to be compressive. This is counterintuitive, as drying failure evidently occurs through tensile cracking.

To address this paradox Scherer (1992) postulated a scenario suggesting that desiccation cracking is necessarily triggered through crack propagation from a tip of a pre-existing structural flaw, at which a substantial total stress concentration occurs. As a result, the remote total stress is substantially magnified through the stress concentration and becomes larger in absolute terms than the elevated suction. In turn, the local effective stress becomes tensile, and hence prone to reach the tensile strength of the material.

Based on the presented results we postulate that a non-symmetric finger of air entry, as described above, constitutes a sufficient imperfection (flaw) in the drying granular body to produce a concentration of total stress induced by the kinematic restraints to the drying shrinkage around the tip of the flaw, to generate crack propagation, as proposed by Péron et al. (2010), and $\mathrm{Hu}$ et al. (2013d), following the idea of Scherer (1992).

In this spirit we consider an air finger (flaw) with depth $c$ and curvature radius at the finger tip of $r c$ at the soil surface, which is surrounded by the micro-tubes pore system, as shown in Fig. 5. The system of micro-tubes representing in the meso-scale the pore system is adapted from the model of $\mathrm{Hu}$ et al. (2013a) to simulate the shrinkage mechanism for silty soil due to water evaporation. In this model, the soil pore structure is approximated by a network of hollow cylinder tubes. The external radius $\left(R_{\text {ext }}\right)$ of the tubes is considered equal to $3.6 \mu \mathrm{m}$ and the current internal radius $\left(R_{\text {int }}\right)$ is equal to $0.78 \mu \mathrm{m}$. These radii are obtained based on the mercury intrusion porosimetry data on Bioley silt (Hu et al. 2013a). Using the principles of linear fracture mechanics, the stress at the external boundary of the tubes which are

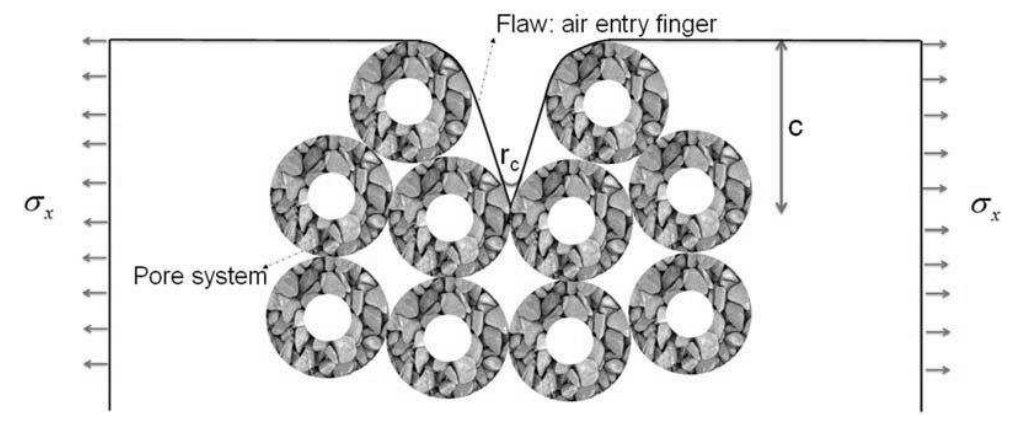

Fig. 5. Idealized meso-scale pore network in the vicinity of an air finger. 
placed near the flaw tip can be calculated. Following the methodology proposed by Scherer (1992), the far-field (macro-scale) stress at crack initiation can be found as (stress is positive in tension)

$$
\sigma_{x}=\frac{K_{\text {Icrit }}+\frac{1}{2} p \sqrt{\pi r_{c}}}{B \sqrt{\pi c}},
$$

where $K_{\text {Icrit }}$ is the critical stress intensity factor, $B$ is a constant equal to 1.12 , and $p$ is the fluid pressure inside of the pore.

The depth of a flaw (c) can be estimated from Fig. 3c as 5 times the diameter of the original biggest pore, assessed for Bioley silt as $d=1.5 \mu \mathrm{m}$ (hence $c=7.5 \mu \mathrm{m}$ for that silt) - Hu et al. (2013a). The flaw tip curvature radius $r_{c}$ is found to be of the same order as the internal radius of the biggest pore $(d / 2=0.375 \mu \mathrm{m})$. A desiccation crack is generally initiated when the soil suction reaches the suction value at the air entry moment. Considering the biggest current pore size of $0.78 \mu \mathrm{m}$, the suction value at air entry for Bioley silt has been calculated as $194 \mathrm{kPa}$ (Hu et al. 2013b). Adopting a value of $0.16 \mathrm{kN} / \mathrm{m}^{3 / 2}$ for the critical stress intensity factor as measured by Chertkov (1995) for a sandy silt soil, a value of $\sigma_{x}=39 \mathrm{kPa}$ is obtained for the macro-scale total stress from Eq. 3. This value is close enough to the one obtained experimentally by Péron (2008) in simulations of the drying experiments on constrained slabs of Bioley silt $(25 \mathrm{kPa})$. The local total stress at the flaw tip is hence estimated as

$$
\sigma_{c}^{\max }=2 B \sigma_{x} \sqrt{\frac{c}{r_{c}}},
$$

which yields a value of $295 \mathrm{kPa}$ (tension).

We shall now evaluate the stress resulting within the tubes imposed by the conditions arising at the tip, as calculated at the meso-scale. For that purpose, we employ a finite element representation of the two tubes at the micro-scale. The tubes are located near the air finger tip in an ideally symmetrical position, as shown in Fig. 3b. The material of the tubes is assumed as linear elastic, with $E=52 \mathrm{MPa}$ and $v=0.3$, following Péron's (2008) data for Bioley silt.

As shown via the numerical simulations, the effective principal stress at failure is equal to $236 \mathrm{kPa}$ (tension), however at the macro-scale this value is $-42 \mathrm{kPa}$ (compression). These differences in sign of the effective stress explain the apparent contradiction of obvious soil shrinkage during the drying process due to compressive effective stress at macro-scale and observing the desiccation cracks which originate from micro-scale by a tensile failure mechanism. 


\section{CONCLUSIONS}

The purpose of the paper is to assess whether air entry fingers may constitute sufficient imperfections in the soil mass to become the cause of a stress concentration and hence result into sufficiently high local tensile effective stress at the air finger-tip. It was found that for the observed geometry of the model-medium and for the values of the drying generated suction in silt this is the case. That demonstrates that the air entry eventually induces crack propagation at the very site of the air entry. The meso-scale linear fracture mechanics analysis yields the stress values in the plausible range of tensile strength.

Acknowledgments. The authors acknowledge the partial support of US NSF grant 0700294, CMMI Div., Geomechanics and Geomaterials Program (T. Hueckel) and support of CNRS, IRSN (MIST laboratory) and the Languedoc-Roussillon Region, France (B. Mielniczuk, M.S. El Youssoufi, T. Hueckel).

\section{References}

Brinker, C.J., and G.W. Scherer (1990), Sol-Gel Science. The Physics and Chemistry of Sol-Gel Processing, Academic Press, San Diego.

Childs, E.C. (1969), An Introduction to the Physical Basis of Soil Water Phenomena, John Wiley-Interscience, London, 493 pp.

Chertkov, V.Y. (1995), Evaluation for soil of crack net connectedness and critical stress-intensity factor, Int. Agrophys. 9, 189-195.

Corte, A., and A. Higashi (1964), Experimental research on desiccation cracks in soil, Final Rep. CRREL-RR-66, National Technical Information Service, Alexandria, USA.

Fredlund, D.G., and H. Rahardjo (1993), Soil Mechanics for Unsaturated Soils, John Wiley \& Sons, New York.

Fung, Y.C. (1984), Biodynamics: Circulation, Springer, New York.

Hu, L.B., H. Péron, T. Hueckel, and L. Laloui (2013a), Desiccation shrinkage of non-clayey soils: multiphysics mechanisms and a microstructural model, Int. J. Numer. Anal. Meth. Geomech. 37, 12, 1761-1781, DOI: 10.1002/ nag.2108.

Hu, L.B., H. Péron, T. Hueckel, and L. Laloui (2013b), Desiccation shrinkage of non-clayey soils: a numerical study, Int. J. Numer. Anal. Meth. Geomech. 37, 12, 1782-1800, DOI: 10.1002/nag.2107.

Hu, L.B., H. Péron, T. Hueckel, and L. Laloui (2013c), Mechanisms and critical properties in drying shrinkage of soils: experimental and numerical parametric studies, Can. Geoech. J. 50, 5, 536-549, DOI: 10.1139/cgj-2012-0065. 
Hu, L.B., M. Monfared, B. Mielniczuk, L. Laloui, T. Hueckel, and M.S. El Youssoufi (2013d), Multi-scale approach to cracking criteria for drying silty soils. In: GeoCongress 2013, 4-7 March 2013, San Diego, USA, 838-845.

Kodikara, J., S.L. Barbour, and D.G. Fredlund (2002), Structure development in surficial heavy clay soils: A synthesis of mechanisms, Aust. Geomech. 37, 3, 25-40.

Lu, N., and W.J. Likos (2004), Unsaturated Soil Mechanics, John Wiley, New York.

Maeda, N., J.N. Israelachvili, and M.M. Kohonen (2003), Evaporation and instabilities of microscopic capillary bridges, Proc. Natl. Acad. Sci. USA 100, 3, 803-808, DOI: 10.1073/pnas.0234283100.

Mielniczuk, B., T. Hueckel, and M.S. El Youssoufi (2013), Micro-scale study of rupture in desiccating granular media. In: GeoCongress 2013, 4-7 March 2013, San Diego, USA.

Mielniczuk, B., M.S. El Youssoufi, L. Sabatier, and T. Hueckel (2014), Rupture of a liquid bridge between two grains due to its evaporation, Acta Geophys. (in revision).

Pellenq, R.J.M., B. Coasne, R.O. Denoyel, and O. Coussy (2009), Simple phenomenological model for phase transitions in confined geometry. 2. Capillary condensation/evaporation in cylindrical mesopores, Langmuir 25, 3, 13931402, DOI: $10.1021 / 1 \mathrm{la} 8020244$.

Péron, H. (2008), Desiccation cracking of soils, Ph.D. Thesis, EPFL, Lausanne.

Péron, H., T. Hueckel, L. Laloui, and L.B. Hu (2009), Fundamentals of desiccation cracking of fine-grained soils: experimental characterisation and mechanisms identification, Can. Geotech. J. 46, 10, 1177-1201, DOI: 10.1139/ T09-054.

Péron, H., L. Laloui, L.B. Hu, and T. Hueckel (2010), Desiccation cracking of soils. In: L. Laloui (ed.), Mechanics of Unsaturated Geomaterials, John Wiley, Hoboken, 55-86.

Péron, H., L. Laloui, L.B. Hu, and T. Hueckel (2013), Formation of drying crack patterns in soils: a deterministic approach, Acta Geotech. 8, 2, 215-221, DOI: $10.1007 / \mathrm{s} 11440-012-0184-5$.

Scherer, G.W. (1992), Crack-tip stress in gels, J. Non-Cryst. Solids 144, 210-216, DOI: $10.1016 / \mathrm{S} 0022-3093(05) 80402-8$.

Taylor, G. (1959), The dynamics of thin sheets of fluid. III. Disintegration of fluid sheets, Proc. Roy. Soc. London A 253, 1274, 313-321, DOI: 10.1098/rspa. 1959.0196.

Terzaghi, K. (1927), Concrete roads: A problem in foundation engineering, J. Boston Soc. Civil Eng. 14, 265-282. 